\title{
SHARP ESTIMATES OF RADIAL MINIMIZERS OF $p$-LAPLACE EQUATIONS
}

\author{
MIGUEL ANGEL NAVARRO AND SALVADOR VILLEGAS
}

\begin{abstract}
In this paper we study semi-stable, radially symmetric and decreasing solutions $u \in W^{1, p}\left(B_{1}\right)$ of $-\Delta_{p} u=g(u)$ in $B_{1} \backslash\{0\}$, where $B_{1}$ is the unit ball of $\mathbb{R}^{N}, p>1, \Delta_{p}$ is the $p$-Laplace operator and $g$ is a general locally Lipschitz function. We establish sharp pointwise estimates for such solutions. As an application of these results, we obtain optimal pointwise estimates for the extremal solution and its derivatives (up to order three) of the equation $-\Delta_{p} u=\lambda f(u)$, posed in $B_{1}$, with Dirichlet data $\left.u\right|_{\partial B_{1}}=0$, where the nonlinearity $f$ is an increasing $C^{1}$ function with $f(0)>0$ and $\lim _{t \rightarrow+\infty} \frac{f(t)}{t^{p-1}}=+\infty$. In addition, we provide, for $N \geq p+4 p /(p-1)$, a large family of semi-stable radially symmetric and decreasing unbounded $W^{1, p}\left(B_{1}\right)$ solutions.
\end{abstract}

\section{INTRODUCTION AND MAIN RESULTS}

This paper is concerned with the semi-stability of radially symmetric and decreasing solutions $u \in W^{1, p}\left(B_{1}\right)$ of

$$
-\Delta_{p} u=g(u) \text { in } B_{1} \backslash\{0\},
$$

where $p>1, \Delta_{p}$ is the $p$-Laplace operator, $B_{1}$ is the unit ball of $\mathbb{R}^{N}$, and $g: \mathbb{R} \longrightarrow \mathbb{R}$ is a locally Lipschitz function.

By abuse of notation, we write $u(r)$ instead of $u(x)$, where $r=|x|$ and $x \in \mathbb{R}^{N}$. We denote by $u_{r}$ the radial derivative of a radial function $u$.

A radial solution $u \in H^{1}\left(B_{1}\right)$ of (1.1) such that $u_{r}(r)<0$ for all $r \in(0,1)$ is called semi-stable if

$$
\int_{B_{1}}(p-1)\left|u_{r}\right|^{p-2}\left|\xi_{r}\right|^{2}-g^{\prime}(u) \xi^{2} \geq 0,
$$

for every radially symmetric function $\xi \in C_{c}^{1}\left(B_{1} \backslash\{0\}\right)$.

Note that the above expression is nothing but the second variation of the energy functional associated to (1.1):

$$
E_{\Omega}(u):=\frac{1}{p} \int_{\Omega}|\nabla u|^{p} d x-\int_{\Omega} G(u) d x
$$

where $G^{\prime}=g$ and $\Omega \subset B_{1}$. Thus, if $u$ is a radial local minimizer of (1.2) with $\Omega=B_{1}$ (i.e., for every $\delta \in(0,1)$ there exists $\varepsilon_{\delta}>0$ such that

The authors have been supported by the MEC Spanish grant MTM2012-37960. 
$E_{B_{1} \backslash \overline{B_{\delta}}}(u) \leq E_{B_{1} \backslash \overline{B_{\delta}}}(u+\xi)$, for all radial functions $\left.\xi \in C_{c}^{1}\left(B_{1} \backslash \overline{B_{\delta}}\right)\right)$ satisfying $\|\xi\|_{C^{1}} \leq \varepsilon_{\delta}$ ), then $u$ is a semi-stable solution of (1.1). Other general situations include semi-stable solutions: for instance, minimal solutions, extremal solutions, and also some solutions between a sub and a supersolution (see [3, Rem. 1.7] for more details). All the results obtained in this paper were obtained by the second author in [16] for the Laplace operator $(p=2)$.

As an application of some general results obtained in this paper for this class of solutions (for arbitrary $g \in C^{1}(\mathbb{R})$ ), we consider the following problem

$$
\left\{\begin{aligned}
-\Delta_{p} u & =\lambda f(u) & & \text { in } B_{1}, \\
u & >0 & & \text { in } B_{1}, \\
u & =0 & & \text { in } \partial B_{1},
\end{aligned}\right.
$$

where $\lambda>0$ and $f$ is an increasing $C^{1}$ function with $f(0)>0$ and

$$
\lim _{t \rightarrow+\infty} \frac{f(t)}{t^{p-1}}=+\infty \text {. }
$$

This problem is studied by Cabré and Sanchón in [4 for general smooth bounded domains $\Omega$ of $\mathbb{R}^{N}$. It is proved that there exists a positive parameter $\lambda^{*}$ such that if $\lambda \in\left(0, \lambda^{*}\right)$ then $\left(1.3_{\lambda, p}\right)$ admits a minimal (smallest) solution $u_{\lambda} \in C^{1}(\bar{\Omega})$ and if $\lambda \in\left(\lambda^{*},+\infty\right)$ then $\left(1.3_{\lambda, p}\right)$ admits no regular solution. In addition, for $\lambda \in\left(0, \lambda^{*}\right)$ the minimal solution $u_{\lambda}$ is semi-stable (in a similar sense of the definition when $\Omega=B_{1}$ ). On the other hand, we may consider the increasing limit

$$
u^{*}:=\lim _{\lambda \uparrow \lambda^{*}} u_{\lambda} .
$$

In the case $p=2$ it is well-known that $u^{*}$ is a weak solution of $\left(1.3_{\lambda, p}\right)$, for $\lambda=\lambda^{*}$. It is called the extremal solution. For general $p, \Omega$ and $f$, it is not known if $u^{*}$ is a weak solution of $\left(1.3_{\lambda, p}\right)$, for $\lambda=\lambda^{*}$. In the case $\Omega=B_{1}$, Cabré, Capella and Sanchón [3] proved that $u^{*}$ is actually a semistable radially decreasing energy solution (i.e. $\left.u^{*} \in W_{0}^{1, p}\right)$ of $\left(1.3_{\lambda, p}\right)$. Hence we can apply to the extremal solution the results obtained in this paper for this kind of solutions.

We refer to [2, 5] for surveys on minimal and extremal solutions and to [1, 6, 7, 8, 9, 10, 12, 13, 14, 15, 17] for other interesting results in the topic of extremal solutions.

The main result obtained in [3] related to the extremal solution of $\left(1.3_{\lambda, p}\right)$ is the following

Theorem 1.1. ([3]). Let $\Omega=B_{1}, p>1$, and that $f$ satisfies (1.4). Let $u^{*}$ be the extremal solution of $\left(1.3_{\lambda, p}\right)$. We have that

i) If $N<p+4 p /(p-1)$, then $u^{*} \in L^{\infty}\left(B_{1}\right)$.

ii) If $N=p+4 p /(p-1)$, then $u^{*}(r) \leq C|\log r|, \forall r \in(0,1)$ and for some constant $C$. 
iii) If $N>p+4 p /(p-1)$, then $u^{*}(r) \leq C r^{-\frac{1}{p}\left(N-2 \sqrt{\frac{N-1}{p-1}}-p-2\right)}|\log r|^{\frac{1}{p}}$, $\forall r \in(0,1)$ and for some constant $C$.

iv) If $N \geq p+4 p /(p-1)$, then $\left|u_{r}^{*}(r)\right| \leq C r^{-\frac{1}{p}\left(N-2 \sqrt{\frac{N-1}{p-1}}-2\right)}|\log r|^{\frac{1}{p}}$, $\forall r \in(0,1)$ and for some constant $C$.

In this paper we establish sharp pointwise estimates for $u^{*}$ and its derivatives (up to order three). We improve the above theorem, answering affirmatively to an open question raised in [3], about the removal of the factor $|\log r|^{\frac{1}{p}}$.

Theorem 1.2. Let $p>1$. Suppose that $f$ satisfies (1.4). Let $u^{*}$ be the extremal solution of $\left(1.3_{\lambda, p}\right)$. We have that

i) If $p \leq N<p+4 p /(p-1)$, then $u^{*}(r) \leq C(1-r), \forall r \in(0,1]$.

ii) If $N=p+4 p /(p-1)$, then $u^{*}(r) \leq C|\log r|, \forall r \in(0,1]$.

iii) If $N>p+4 p /(p-1)$, then

$$
u^{*}(r) \leq C\left(r^{-\frac{1}{p}\left(N-2 \sqrt{\frac{N-1}{p-1}}-p-2\right)}-1\right), \forall r \in(0,1] .
$$

iv) If $N \geq p+4 p /(p-1)$, then

$$
\left|\partial_{r}^{(k)} u^{*}(r)\right| \leq C r^{-\frac{1}{p}\left(N-2 \sqrt{\frac{N-1}{p-1}}+(k-1) p-2\right)}, \forall r \in(0,1], \forall k \in\{1,2\} .
$$

v) If $N \geq p+4 p /(p-1)$, and $f$ is convex, then

$$
\left|u_{r r r}^{*}(r)\right| \leq C r^{-\frac{1}{p}\left(N-2 \sqrt{\frac{N-1}{p-1}}+2 p-2\right)}, \forall r \in(0,1] .
$$

Where $C=C_{N, p} \min _{t \in[1 / 2,1]}\left|u_{r}(t)\right|$, and $C_{N, p}$ is a constant depending only on $N$ and $p$.

Remark 1.3. In 11 García-Azorero, Peral and Puel proved that if $f(u)=$ $e^{u}$ and $N=p+4 p /(p-1)$ then

$$
u^{*}(r)=-p \log r \text { and } \lambda^{*}=4 p^{p} /(p-1) .
$$

This shows that the pointwise estimates of Theorem 1.2 are optimal for $N=p+4 p /(p-1)$.

On the other hand, in [3] Cabré and Sanchón proved that if $N>p+$ $4 p /(p-1)$ and $f(u)=(1+u)^{m}$, where

$$
m:=\frac{(p-1) N-2 \sqrt{(p-1)(N-1)}-p+2}{N-2 \sqrt{\frac{N-1}{p-1}}-p-2},
$$


then

$$
u^{*}(r)=r^{-\frac{1}{p}\left(N-2 \sqrt{\frac{N-1}{p-1}}-p-2\right)}-1
$$

and

$$
\lambda^{*}=\left(\frac{p}{m-(p-1)}\right)^{p-1}\left(N-\frac{m p}{m-(p-1)}\right) .
$$

This also shows the optimality of the pointwise estimates of Theorem 1.2 for the case $N>p+4 p /(p-1)$.

As mentioned before, the proof of Theorem 1.2 is based on general properties of semi-stable radially decreasing energy solutions. Our main results about these type of solutions are the following.

Theorem 1.4. Let $p>1, g: \mathbb{R} \longrightarrow \mathbb{R}$ be a locally Lipschitz function, and $u \in W^{1, p}\left(B_{1}\right)$ be a semi-stable radial solution of (1.1) satisfying $u_{r}(r)<0$ for all $r \in(0,1)$. Then there exists a constant $C_{N, p}$ depending only on $N$ and $p$ such that:

i) If $p \leq N<p+4 p /(p-1)$, then $\|u\|_{L^{\infty}\left(B_{1}\right)} \leq C_{N, p}\|u\|_{W^{1, p}\left(B_{1} \backslash \overline{B_{1 / 2}}\right)}$.

ii) If $N=p+4 p /(p-1)$, then

$$
|u(r)| \leq C_{p+4 p /(p-1), p}\|u\|_{W^{1, p}\left(B_{1} \backslash \overline{B_{1 / 2}}\right)}(|\log r|+1), \forall r \in(0,1] .
$$

iii) If $N>p+4 p /(p-1)$, then

$$
|u(r)| \leq C_{N, p}\|u\|_{W^{1, p}\left(B_{1} \backslash \overline{B_{1 / 2}}\right)} r^{-\frac{1}{p}\left(N-2 \sqrt{\frac{N-1}{p-1}}-p-2\right)}, \forall r \in(0,1] .
$$

Theorem 1.5. Let $N \geq p+4 p /(p-1), g: \mathbb{R} \longrightarrow \mathbb{R}$ be a locally Lipschitz function, and $u \in W^{1, p}\left(B_{1}\right)$ be a semi-stable radial solution of (1.1) satisfying $u_{r}(r)<0$ for all $r \in(0,1)$. Then there exists a constant $C_{N, p}^{\prime}$ depending only on $N$ and $p$ such that:

i) If $g \geq 0$, then

$$
\left|u_{r}(r)\right| \leq C_{N, p}^{\prime}\|\nabla u\|_{L^{p}\left(B_{1} \backslash B_{1 / 2}\right)^{r}} r^{-\frac{1}{p}\left(N-2 \sqrt{\frac{N-1}{p-1}}-2\right)}, \forall r \in(0,1 / 2] .
$$

ii) If $g \geq 0$ is nondecreasing, then

$$
\left|u_{r r}(r)\right| \leq C_{N, p}^{\prime}\|\nabla u\|_{L^{p}\left(B_{1} \backslash B_{1 / 2}\right)} r^{-\frac{1}{p}\left(N-2 \sqrt{\frac{N-1}{p-1}}+p-2\right)}, \forall r \in(0,1 / 2] .
$$

iii) If $g \geq 0$ is nondecreasing and convex, then

$$
\left|u_{r r r}(r)\right| \leq C_{N, p}^{\prime}\|\nabla u\|_{L^{p}\left(B_{1} \backslash B_{1 / 2}\right)} r^{-\frac{1}{p}\left(N-2 \sqrt{\frac{N-1}{p-1}}+2 p-2\right)}, \forall r \in(0,1 / 2] .
$$


Remark 1.6. We emphasize that the estimates obtained in Theorems 1.4 and 1.5 are in terms of the $W^{1, p}$ norm of the annulus $B_{1} \backslash \overline{B_{1 / 2}}$, while $u$ is required to belong to $W^{1, p}\left(B_{1}\right)$. In fact, this requirement is essential to obtain our results, since we can easily find semi-stable radially decreasing solutions of (1.1) (for instance $u(r)=r^{-s}-1$, with s large enough), not in the energy class $W^{1, p}\left(B_{1}\right)$, for which the statements of Theorems 1.4 and 1.5 fail to satisfy.

Remark 1.7. To our knowledge there is no estimates of $\left|u_{r r}\right|$ or $\left|u_{r r r}\right|$ in the literature for this kind of solutions. Moreover, we prove that without assumptions on the sign of $g, g^{\prime}$ or $g^{\prime \prime}$ it is not possible to obtain any pointwise estimate for $\left|u_{r}\right|,\left|u_{r r}\right|$ or $\left|u_{r r r}\right|$ (see Corollaries 3.4, 3.6 and 3.9).

\section{Proof of the main Results}

Lemma 2.1. Let $N \geq p>1, g: \mathbb{R} \longrightarrow \mathbb{R}$ be a locally Lipschitz function, and $u \in W^{1, p}\left(B_{1}\right)$ be a semi-stable radial solution of (1.1) satisfying $u_{r}(r)<0$ for all $r \in(0,1)$. Then there exists a constant $K_{N, p}$ depending only on $N$ and $p$ such that:

$$
\int_{0}^{r}\left|u_{r}(t)\right|^{p} t^{N-1} d t \leq K_{N, p}\|\nabla u\|_{L^{p}\left(B_{1} \backslash B_{1 / 2}\right)}^{p} r^{2 \sqrt{\frac{N-1}{p-1}}+2}, \forall r \in[0,1] .
$$

Proof. Let us use [3, Lem. 2.2] (see also the proof of [3, Lem. 2.3]) to assure that

$$
(N-1) \int_{B_{1}}\left|u_{r}\right|^{p} \eta^{2} d x \leq(p-1) \int_{B_{1}}\left|u_{r}\right|^{p}|\nabla(|x| \eta)|^{2} d x
$$

for every radial Lipschitz function $\eta$ vanishing on $\partial B_{1}$.

We now fix $r \in(0,1 / 2)$ and consider the function

$$
\eta(t)= \begin{cases}r^{-\sqrt{\frac{N-1}{p-1}}-1} & , \text { if } 0 \leq t \leq r \\ t^{-\sqrt{\frac{N-1}{p-1}}-1} & , \text { if } r<t \leq 1 / 2, \\ 2^{\sqrt{\frac{N-1}{p-1}}+2}(1-t) & , \text { if } 1 / 2<t \leq 1 .\end{cases}
$$

Let $v(t)=(N-1) \eta(t)^{2}-(p-1)(t \eta(t))^{\prime 2}$. Since $v(t)=0$ for $r<t \leq 1 / 2$, inequality (2.6) shows that

$$
\begin{aligned}
(N-p) r^{-2 \sqrt{\frac{N-1}{p-1}}-2} \int_{0}^{r}\left|u_{r}(t)\right|^{p} t^{N-1} d t & \leq-\int_{1 / 2}^{1} v(t)\left|u_{r}(t)\right|^{p} t^{N-1} d t \\
& \leq \alpha_{N, p} \int_{1 / 2}^{1}\left|u_{r}(t)\right|^{p} t^{N-1} d t
\end{aligned}
$$

where the constant $\alpha_{N, p}=\max _{1 / 2 \leq t \leq 1}-v(t)$ dependes only on $N$ and $p$. This establishes (2.5) for $r \in[0,1 / 2]$, if $N>p$. 
If $r \in(1 / 2,1]$ and $N>p$ then, applying the above inequality for $r=1 / 2$, we obtain

$$
\begin{array}{r}
\int_{0}^{r}\left|u_{r}(t)\right|^{p} t^{N-1} d t \leq \int_{0}^{1 / 2}\left|u_{r}(t)\right|^{p} t^{N-1} d t+\int_{1 / 2}^{1}\left|u_{r}(t)\right|^{p} t^{N-1} d t \\
\leq\left[\frac{\alpha_{N, p}}{N-p}\left(\frac{1}{2}\right)^{2 \sqrt{\frac{N-1}{p-1}}+2}+1\right] \int_{1 / 2}^{1}\left|u_{r}(t)\right|^{p} t^{N-1} d t \\
\leq(2 r)^{2 \sqrt{\frac{N-1}{p-1}}+2}\left[\frac{\alpha_{N, p}}{N-p}\left(\frac{1}{2}\right)^{2 \sqrt{\frac{N-1}{p-1}}+2}+1\right] \int_{1 / 2}^{1}\left|u_{r}(t)\right|^{p} t^{N-1} d t
\end{array}
$$

which is the desired conclusion with $K_{N, p}=\frac{1}{\omega_{N}}\left(\frac{\alpha_{N, p}}{N-p}+2^{2 \sqrt{\frac{N-1}{p-1}}+2}\right)$.

Finally, if $N=p$, changing the definition of $\eta(t)$ in $[0, r]$ by

$$
\eta(t)= \begin{cases}\frac{r^{-\sqrt{\frac{N-1}{p-1}}}}{r_{0}} & , \text { if } 0 \leq t \leq r_{0}, \\ \frac{r^{-\sqrt{\frac{N-1}{p-1}}}}{t} & , \text { if } r_{0}<t \leq r,\end{cases}
$$

for arbitrary $r_{0} \in(0, r)$, we obtain

$$
(N-1) r^{-2 \sqrt{\frac{N-1}{p-1}}-2} \int_{r_{0}}^{r}\left(\frac{r}{t}\right)^{2}\left|u_{r}(t)\right|^{p} t^{N-1} d t \leq C_{N, p} \int_{1 / 2}^{1}\left|u_{r}(t)\right|^{p} t^{N-1} d t .
$$

Letting $r_{0} \rightarrow 0$ and taking into account that $r / t \geq 1$ for $0<t \leq r$ yields (2.5) for $N=p$ and $r \in[0,1 / 2]$. If $r \in(1 / 2,1]$, we apply similar arguments to the case $N>p$ to complete the proof.

Proposition 2.2. Let $N \geq p>1, g: \mathbb{R} \longrightarrow \mathbb{R}$ be a locally Lipschitz function, and $u \in W^{1, p}\left(B_{1}\right)$ be a semi-stable radial solution of (1.1) satisfying $u_{r}(r)<0$ for all $r \in(0,1)$. Then there exists a constant $K_{N, p}^{\prime}$ depending only on $N$ and $p$ such that:

$$
\left|u(r)-u\left(\frac{r}{2}\right)\right| \leq K_{N, p}^{\prime}\|\nabla u\|_{L^{p}\left(B_{1} \backslash B_{1 / 2}\right)} r^{-\frac{1}{p}\left(N-2 \sqrt{\frac{N-1}{p-1}}-p-2\right)}, \forall r \in(0,1] .
$$


Proof. Fix $r \in(0,1]$. Applying Hölder's inequality and Lemma 2.1 we deduce

$$
\begin{aligned}
& \left|u(r)-u\left(\frac{r}{2}\right)\right|=\int_{r / 2}^{r}\left|u_{r}(t)\right| t^{\frac{N-1}{p}} \frac{1}{t^{\frac{N-1}{p}}} d t \\
& \leq\left(\int_{r / 2}^{r}\left|u_{r}(t)\right|^{p} t^{N-1} d t\right)^{\frac{1}{p}}\left(\int_{r / 2}^{r} t^{-\frac{(N-1) p^{\prime}}{p}} d t\right)^{\frac{1}{p^{\prime}}}
\end{aligned}
$$

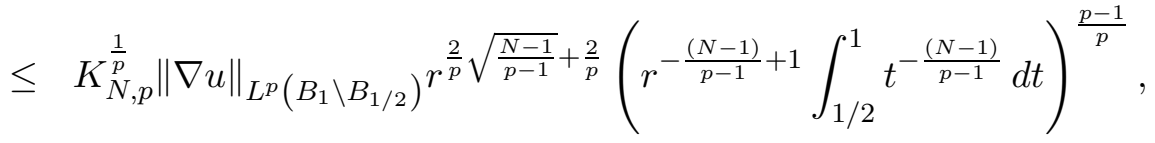

and (2.7) is proved.

Proof of the Theorem 1.4. Let $0<r \leq 1$. Then, there exist $m \in \mathbb{N}$ and $1 / 2<r_{1} \leq 1$ such that $r=r_{1} / 2^{m-1}$. Since $u$ is radial we have $u\left(r_{1}\right) \leq$ $\|u\|_{L^{\infty}\left(B_{1} \backslash \overline{B_{1 / 2}}\right)} \leq \gamma_{N, p}\|u\|_{W^{1, p}\left(B_{1} \backslash \overline{B_{1 / 2}}\right)}$, where $\gamma_{N, p}$ dependes only on $N$ and $p$. From this an Proposition 2.2, it follows that

$$
\begin{aligned}
|u(r)| \leq & \left|u(r)-u\left(r_{1}\right)\right|+\left|u\left(r_{1}\right)\right| \\
= & \sum_{i=1}^{m-1}\left|u\left(\frac{r_{1}}{2^{i-1}}\right)-u\left(\frac{r_{1}}{2^{i}}\right)\right|+\left|u\left(r_{1}\right)\right| \\
\leq & K_{N, p}^{\prime}\|\nabla u\|_{L^{p}\left(B_{1} \backslash B_{1 / 2}\right)} \sum_{i=1}^{m-1}\left(\frac{r_{1}}{2^{i-1}}\right)^{-\frac{1}{p}\left(N-2 \sqrt{\frac{N-1}{p-1}}-p-2\right)} \\
& +\gamma_{N, p}\|u\|_{W^{1, p}\left(B_{1} \backslash \overline{B_{1 / 2}}\right)} \leq \\
\leq & \left.\left(K_{N, p}^{\prime} \sum_{i=1}^{m-1}\left(\frac{r_{1}}{2^{i-1}}\right)^{-\frac{1}{p}\left(N-2 \sqrt{\frac{N-1}{p-1}}-p-2\right)}+\gamma_{N, p}\right)\|u\|_{W^{1, p}\left(B_{1} \backslash \overline{B_{1 / 2}}\right.}\right)
\end{aligned}
$$

- If $p \leq N<p+4 p /(p-1)$, we have $-\frac{1}{p}\left(N-2 \sqrt{\frac{N-1}{p-1}}-p-2\right)>0$.

Then

$$
\sum_{i=1}^{m-1}\left(\frac{r_{1}}{2^{i-1}}\right)^{-\frac{1}{p}\left(N-2 \sqrt{\frac{N-1}{p-1}}-p-2\right)} \leq \sum_{i=1}^{\infty}\left(\frac{1}{2^{i-1}}\right)^{-\frac{1}{p}\left(N-2 \sqrt{\frac{N-1}{p-1}}-p-2\right)}
$$

which is a convergent series.

- If $N=p+4 p /(p-1)$, we have $-\frac{1}{p}\left(N-2 \sqrt{\frac{N-1}{p-1}}-p-2\right)=0$. From (2.8) we obtain

$$
\begin{aligned}
|u(r)| & \leq\left[K_{N, p}^{\prime}(m-1)+\gamma_{N, p}\right]\|u\|_{W^{1, p}\left(B_{1} \backslash \overline{B_{1 / 2}}\right)} \\
& \left.=\left[K_{N, p}^{\prime}\left(\frac{\log r_{1}-\log r}{\log 2}\right)+\gamma_{N, p}\right]\|u\|_{W^{1, p}\left(B_{1} \backslash \overline{B_{1 / 2}}\right.}\right) \\
& \leq\left(\frac{K_{N, p}^{\prime}}{\log 2}+\gamma_{N, p}\right)(|\log r|+1)\|u\|_{W^{1, p}\left(B_{1} \backslash \overline{B_{1 / 2}}\right)},
\end{aligned}
$$


- If $N>p+4 p /(p-1)$, we have $-\frac{1}{p}\left(N-2 \sqrt{\frac{N-1}{p-1}}-p-2\right)<0$. Then

$$
\sum_{i=1}^{m-1}\left(\frac{r_{1}}{2^{i-1}}\right)^{-\frac{1}{p}\left(N-2 \sqrt{\frac{N-1}{p-1}}-p-2\right)}=\frac{r^{-\frac{1}{p}\left(N-2 \sqrt{\frac{N-1}{p-1}}-p-2\right)}-r_{1}^{-\frac{1}{p}\left(N-2 \sqrt{\frac{N-1}{p-1}}-p-2\right)}}{(1 / 2)^{-\frac{1}{p}\left(N-2 \sqrt{\frac{N-1}{p-1}}-p-2\right)}-1} .
$$

From (2.8), we conclude

$$
|u(r)| \leq\left(\frac{K_{N, p}^{\prime}}{(1 / 2)^{-\frac{1}{p}\left(N-2 \sqrt{\frac{N-1}{p-1}}-p-2\right)}-1}+\gamma_{N, p}\right) r^{-\frac{1}{p}\left(N-2 \sqrt{\frac{N-1}{p-1}}-p-2\right)}\|u\|_{W^{1, p}\left(B_{1} \backslash \overline{B_{1 / 2}}\right)},
$$

which completes the proof.

Lemma 2.3. Let $N \geq 1, p>1, g: \mathbb{R} \longrightarrow \mathbb{R}$ be a nonnegative and nondecreasing locally Lipschitz function, and $u \in W^{1, p}\left(B_{1}\right)$ be a semi-stable radial solution of (1.1) such that $u_{r}<0$ for all $r \in(0,1)$. Then

$$
g(u(r)) \leq N \frac{\left|u_{r}\right|^{p-1}}{r}, \quad \forall r \in(0,1]
$$

Moreover, if $g$ convex then

$$
g^{\prime}(u(r)) \leq M_{N, p} \frac{\left|u_{r}(r)\right|^{p-2}}{r^{2}}, \quad \forall r \in(0,1],
$$

where $M_{N, p}$ is a constant depending only on $N$ and $p$.

Proof. Consider the function

$$
\Psi(r):=-N r^{1-1 / N}\left|u_{r}\left(r^{1 / N}\right)\right|^{p-2} u_{r}\left(r^{1 / N}\right), r \in(0,1] .
$$

It is easy to check to that $\Psi^{\prime}(r)=g\left(u\left(r^{1 / N}\right)\right), r \in(0,1]$. As $g$ in nonnegative and nondecreasing we have that $\Psi$ is a nonnegative nondecreasing concave function. It follows immediately that

$$
0 \leq \Psi^{\prime}(r) \leq \Psi(r) / r, r \in(0,1],
$$

and we obtain (2.9).

To obtain $i i$ ), we first observe that from (1.1) it is obtained

$$
u_{r r}=-\frac{1}{p-1}\left(\frac{g(u)}{\left|u_{r}\right|^{p-2}}+\frac{N-1}{r} u_{r}\right), \quad \forall r \in(0,1] .
$$

Therefore, using the nonnegativeness of $g$ and (2.9) we deduce that

$$
\left|u_{r r}\right| \leq \frac{1}{p-1}\left(\frac{g(u)}{\left|u_{r}\right|^{p-2}}+\frac{N-1}{r}\left|u_{r}\right|\right) \leq\left(\frac{2 N-1}{p-1}\right) \frac{\left|u_{r}\right|}{r}, \quad \forall r \in(0,1] .
$$

For fixed $\alpha \in \mathbb{R}$ an easy computation shows that

$$
\begin{aligned}
\partial_{r}\left(r^{\alpha}\left|u_{r}\right|^{p-2}\right) & =\alpha r^{\alpha-1}\left|u_{r}\right|^{p-2}-(p-2) r^{\alpha} u_{r r}\left|u_{r}\right|^{p-3} \\
& \geq r^{\alpha-1}\left|u_{r}\right|^{p-2}\left(\alpha-\frac{|p-2|(2 N-1)}{p-1}\right), \quad \forall r \in(0,1] .
\end{aligned}
$$


Thus $r^{\alpha}\left|u_{r}\right|^{p-2}$ is nondecreasing for $\alpha=\frac{|p-2|(2 N-1)}{p-1}$. Using this, the monotocity of $g^{\prime}(u(r))$ and the semi-stability of $u$, we deduce that

$$
\begin{aligned}
g^{\prime}(u(r)) \int_{0}^{r} s^{N-1} \xi(s)^{2} d s & \leq \int_{0}^{r} s^{N-1} g^{\prime}(u(s)) \xi(s)^{2} d s \\
& \leq(p-1) \int_{0}^{r}\left|u_{r}(s)\right|^{p-2} s^{\alpha} s^{N-1-\alpha} \xi^{\prime}(s)^{2} d s \\
& \leq(p-1)\left|u_{r}(r)\right|^{p-2} r^{\alpha} \int_{0}^{r} s^{N-1-\alpha} \xi^{\prime}(s)^{2} d s,
\end{aligned}
$$

for every $r \in(0,1)$ and every $\xi \in C^{1}$ with compact support in $(0, r)$.

Taking $\xi(s)=\zeta\left(\frac{s}{r}\right)$ for $s \in[0, r]$, where $\zeta \in C^{1}$ is any function with compact support in $(0,1)$, we obtain (2.10).

Proof of the Theorem 1.5.

i) We first observe that $\partial_{r}\left(-r^{N-1}\left|u_{r}\right|^{p-2} u_{r}\right)=r^{N-1} g(u)$. Hence $-r^{N-1}\left|u_{r}\right|^{p-2} u_{r}$ is positive nondecreasing function and so is $\left(-r^{N-1}\left|u_{r}\right|^{p-2} u_{r}\right)^{\frac{p}{p-1}}$.

Thus, for $0<r \leq 1 / 2$, we have

$$
\begin{aligned}
\int_{0}^{2 r}\left|u_{r}(t)\right|^{p} t^{N-1} d t & \geq \int_{r}^{2 r}\left|u_{r}(t)\right|^{p} t^{N-1} d t \\
& =\int_{r}^{2 r}\left(-t^{N-1}\left|u_{r}\right|^{p-2} u_{r}\right)^{\frac{p}{p-1}} t^{N-\frac{p(N-1)}{p-1}-1} d t \\
& \geq r^{\frac{p(N-1)}{p-1}}\left|u_{r}(r)\right|^{p} \int_{r}^{2 r} t^{N-\frac{p(N-1)}{p-1}-1} d t \\
& =r^{N}\left|u_{r}(r)\right|^{p} \int_{1}^{2} t^{-\frac{N-1}{p-1}} d t,
\end{aligned}
$$

from this and Lemma 2.1 we obtain $i$ ).

ii) Since (2.13) and $i$ ) it follows $i i$ ).

iii) From (1.1) we obtain

$$
u_{r r r}=-\frac{1}{p-1}\left(\frac{g^{\prime}(u) u_{r}}{\left|u_{r}\right|^{p-2}}-(p-2) \frac{u_{r} u_{r r} g(u)}{\left|u_{r}\right|^{p}}-\frac{N-1}{r^{2}} u_{r}+\frac{N-1}{r} u_{r r}\right) \text {, }
$$

for every $r \in(0,1)$. Therefore from (2.9), (2.10) and (2.13), we obtain

$$
\begin{aligned}
\left|u_{r r r}\right| & \leq \frac{1}{p-1}\left(\frac{g^{\prime}(u)\left|u_{r}\right|}{\left|u_{r}\right|^{p-2}}+|p-2| \frac{\left|u_{r}\right|\left|u_{r r}\right| g(u)}{\left|u_{r}\right| p}+\frac{N-1}{r^{2}}\left|u_{r}\right|+\frac{N-1}{r}\left|u_{r r}\right|\right) \\
& \leq \frac{1}{p-1}\left(M_{N, p}+\frac{N(2 N-1)|p-2|}{p-1}+(N-1)+\frac{(N-1)(2 N-1)}{p-1}\right) \frac{\left|u_{r}\right|}{r^{2}},
\end{aligned}
$$

for every $r \in(0,1]$, and $i i i)$ follows from $i)$. 
Lemma 2.4. Let $N \geq 1, p>1, g: \mathbb{R} \longrightarrow \mathbb{R}$ be a locally Lipschitz nonnegative and nondecreasing function and $u$ be a radial solution of (1.1) satisfying $u_{r}(r)<0$ for all $r \in(0,1)$. Then

i) $r^{N-1}\left|u_{r}\right|^{p-1}$ is nondecreasing for $r \in(0,1]$.

ii) $r^{-1}\left|u_{r}\right|^{p-1}$ is nonincreasing for $r \in(0,1]$.

iii) $\max _{t \in[1 / 2,1]}\left|u_{r}(t)\right| \leq 2^{\frac{N}{p-1}} \min _{t \in[1 / 2,1]}\left|u_{r}(t)\right|$.

iv) $\|\nabla u\|_{L^{p}\left(B_{1} \backslash B_{1 / 2}\right)} \leq q_{N, p} \min _{t \in[1 / 2,1]}\left|u_{r}(t)\right|$ for a certain constant $q_{N, p}$ depending only on $N$ and $p$.

Proof.

i) Since $u_{r}<0$ we have $\partial_{r}\left(r^{N-1}\left|u_{r}\right|^{p-1}\right)=r^{N-1} g(u) \geq 0$

ii) As we have observed in the proof of Lemma 2.3, the function $\Psi(r)=$ $-N r^{1-1 / N}\left|u_{r}\left(r^{1 / N}\right)\right|^{p-2} u_{r}\left(r^{1 / N}\right)$ is nonnegative, nondecreasing and concave for $r \in(0,1]$. Therefore $\Psi(r) / r$ is nonincreasing, and $i i)$ follows immediately.

iii) Take $r_{1}, r_{2} \in[1 / 2,1]$ such that $\left|u_{r}\left(r_{1}\right)\right|=\min _{t \in[1 / 2,1]}\left|u_{r}(t)\right|$ and $\left|u_{r}\left(r_{2}\right)\right|=\max _{t \in[1 / 2,1]}\left|u_{r}(t)\right|$.

-If $r_{2} \leq r_{1}$, we deduce from $\left.i\right)$ that $\left|u_{r}\left(r_{2}\right)\right|^{p-1} \leq\left(r_{1} / r_{2}\right)^{N-1}\left|u_{r}\left(r_{1}\right)\right|^{p-1} \leq$ $2^{N}\left|u_{r}\left(r_{1}\right)\right|^{p-1}$.

-If $r_{2}>r_{1}$, we deduce from $\left.i i\right)$ that $\left|u_{r}\left(r_{2}\right)\right|^{p-1} \leq\left(r_{2} / r_{1}\right)\left|u_{r}\left(r_{1}\right)^{p-1}\right| \leq$ $2\left|u_{r}\left(r_{1}\right)\right|^{p-1} \leq 2^{N}\left|u_{r}\left(r_{1}\right)\right|^{p-1}$.

iv) We see at once that

$$
\|\nabla u\|_{L^{p}\left(B_{1} \backslash B_{1 / 2}\right)} \leq\left|B_{1} \backslash B_{1 / 2}\right|^{1 / p} \max _{t \in[1 / 2,1]}\left|u_{r}(t)\right|
$$

Proof of Theorem 1.2. As we have mentioned, it is well known that $u^{*}$ is a semi-stable radially decreasing $W^{1, p}\left(B_{1}\right)$ solution of (1.1) for $g(s)=\lambda f(s)$. Hence, we can apply to $u^{*}$ the results obtained in Theorems 1.4 and 1.5 and Lemma 2.4.

Let us first prove $i), i i)$, and $i i i)$ for $r \in(0,1 / 2)$. Since $u^{*}(1)=0$, and on account of statement $i v$ ) of Lemma 2.4, we have

$$
\left\|u^{*}\right\|_{W^{1, p}\left(B_{1} \backslash \overline{B_{1 / 2}}\right)} \leq h_{N, p}\left\|\nabla u^{*}\right\|_{L^{p}\left(B_{1} \backslash B_{1 / 2}\right)} \leq h_{N, p}^{\prime} \min _{t \in[1 / 2,1]}\left|u_{r}^{*}(t)\right|,
$$

for certain constants $h_{N, p}, h_{N, p}^{\prime}$ depending only on $N$ and $p$. From this and Theorem 1.4 .

i) follows from the inequality $1 \leq 2(1-r)$, for $r \in(0,1 / 2)$.

ii) follows from the inequality $|\log r|+1 \leq\left(\frac{\log 2+1}{\log 2}\right)|\log r|$, for $r \in$ $(0,1 / 2)$.

iii) follows from the inequality

$$
r^{-\frac{1}{p}\left(N-2 \sqrt{\frac{N-1}{p-1}}-p-2\right)} \leq\left(\frac{(1 / 2)^{-\frac{1}{p}\left(N-2 \sqrt{\frac{N-1}{p-1}}-p-2\right)}}{(1 / 2)^{-\frac{1}{p}\left(N-2 \sqrt{\frac{N-1}{p-1}}-p-2\right)}-1},\right)\left(r^{-\frac{1}{p}\left(N-2 \sqrt{\frac{N-1}{p-1}}-p-2\right)}-1\right)
$$


for $r \in(0,1 / 2)$.

We next show $i), i i)$, and $i i i)$ for $r \in[1 / 2,1]$. From statement $i i i)$ of Lemma 2.4 it follows that

$$
u^{*}(r)=\int_{r}^{1}\left|u^{*}(t)\right| d t \leq(1-r) 2^{\frac{N}{p-1}} \min _{t \in[1 / 2,1]}\left|u_{r}^{*}(t)\right|, \forall r \in[1 / 2,1],
$$

which is the desired conclusion if $N \leq p+4 p /(p-1)$. If $N=p+4 p /(p-1)$, our claim follows from the inequality $1-r \leq|\log r|$, for $r \in[1 / 2,1]$. Finally, if $N>p+4 p /(p-1)$, the desired conclusion follows immediately from the inequality $1-r \leq z_{N, p}\left(r^{-\frac{1}{p}\left(N-2 \sqrt{\frac{N-1}{p-1}}-p-2\right)}-1\right)$, for $r \in[1 / 2,1]$ and certain constant $z_{n, p}>0$.

We now prove statement $i v)$. In the case $k=1$ and $r \in(0,1 / 2)$, it follows immediatety from statement $i$ ) of Theorem 1.5 and statement $i v$ ) of Lemma 2.4. The case $k=1$ and $r \in[1 / 2,1]$ is also obvious on account of statement iii) of Lemma 2.4 and inequality $1 \leq r^{-\frac{1}{p}\left(N-2 \sqrt{\frac{N-1}{p-1}}-p-2\right)}$, for $r \in[1 / 2,1]$, for $N \geq p+4 p /(p-1)$. Finally, as in the proof of statement $i i)$ and $i i i)$ for $f$ convex, of Theorem 1.5, we have

$$
\left|u_{r r}^{*}(r)\right| \leq\left(\frac{2 N-1}{p-1}\right) \frac{\left|u_{r}^{*}(r)\right|}{r},
$$

and

$$
\left|u_{r r r}^{*}(r)\right| \leq s_{N, p} \frac{\left|u_{r}^{*}(r)\right|}{r^{2}}
$$

for $r \in(0,1]$ and certain constant $s_{n, p}>0$., which gives statement $\left.i v\right)$ and $v$ ) from the case $k=1$.

\section{A FAMily OF SEMI-STABle SOlUtions}

Theorem 3.1. Let $h \in\left(C^{2} \cap L^{1}\right)(0,1]$ be a nonnegative function and consider

$$
\Phi(r)=r^{2 \sqrt{\frac{N-1}{p-1}}}\left(1+\int_{0}^{r} h(s) d s\right) \forall r \in(0,1] .
$$

Define $u_{r}<0$ by

$$
\Phi^{\prime}(r)=(N-1) r^{N-3}\left|u_{r}(r)\right|^{p} \forall r \in(0,1] .
$$

Then, for $N \geq p+4 p /(p-1)$, u is a semi-stable radially decreasing unbounded $W^{1, p}\left(B_{1}\right)$ solution of a problem of the type (1.1), where $u$ is any function with radial derivative $u_{r}$.

To prove this theorem, we will use the following lemma, which is a generalization of the classical Hardy inequality:

Lemma 3.2. [16] Let $\Phi \in C^{1}(0, L), 0<L \leq \infty$, satisfying $\Phi^{\prime}>0$. Then

$$
\int_{0}^{L} \frac{4 \Phi^{2}}{\Phi^{\prime}} \xi^{\prime 2} \geq \int_{0}^{L} \Phi^{\prime} \xi^{2}
$$


for every $\xi \in C_{c}^{\infty}(0, L)$.

Proof of the Theorem 1.4. First of all, since $\Phi \in C^{1}(0,1] \cap C[0,1]$ is an increasing function, we obtain $\Phi^{\prime} \in L^{1}(0,1)$ and hence $r^{N-1}\left|u_{r}(r)\right|^{p}=$ $r^{2} \Phi^{\prime}(r) /(N-1) \in L^{1}(0,1)$, which gives $u \in W^{1, p}\left(B_{1}\right)$.

On the other hand, since $\Phi^{\prime}(r) \geq 2 \sqrt{\frac{N-1}{p-1}} r^{2 \sqrt{\frac{N-1}{p-1}}-1}, r \in(0,1]$, we deduce

$$
\left|u_{r}(r)\right| \geq\left(\frac{2}{N-1} \sqrt{\frac{N-1}{p-1}}\right)^{\frac{1}{p}} r^{-\frac{1}{p}\left(N-2 \sqrt{\frac{N-1}{p-1}}-2\right)} .
$$

As $N \geq p+4 p /(p-1)$, we have $-\frac{1}{p}\left(N-2 \sqrt{\frac{N-1}{p-1}}-2\right) \leq-1$. It follows that $u_{r} \notin L^{1}(0,1)$, and since $u$ is radially decreasing, we obtain $\lim _{r \rightarrow 0} u(r)=$ $+\infty$.

Since $h \in C^{2}(0,1]$, it follows that $u_{r} \in C^{2}(0,1]$. Therefore, $\Delta_{p} u \in$ $C^{1}\left(\overline{B_{1}} \backslash\{0\}\right)$. Hence, taking $g \in C^{1}(\mathbb{R})$ such that $g(s)=-\Delta_{p} u\left(u^{-1}(s)\right)$, for $s \in[u(1),+\infty)$, we conclude that $u$ is solution of a problem of the type (1.1).

It remains to prove that $u$ is semi-stable. Taking into account that $u_{r} \neq 0$ in $(0,1]$ and applying [3, Lem. 2.2], the semi-stability of $u$ is equivalent to

$$
(p-1) \int_{0}^{1} r^{N-1}\left|u_{r}\right|^{p} \xi^{2} d x \geq(N-1) \int_{0}^{1} r^{N-3}\left|u_{r}\right|^{p} \xi^{2} d x
$$

for every $\xi \in C_{c}^{\infty}(0,1)$.

For this purpose, we will apply the Lemma 3.2 above. From the definition of $\Phi$, it is easily seen that $\Phi^{\prime}(r) \geq 2 \sqrt{\frac{N-1}{p-1}} \frac{\Phi}{r}, r \in(0,1]$ It follows that

$$
\left(\frac{p-1}{N-1}\right) r^{2} \Phi^{\prime} \geq \frac{4 \Phi^{2}}{\Phi^{\prime}} \text { in }(0,1] \text {. }
$$

Finally, since $\Phi^{\prime}(r)=(N-1) r^{N-3}\left|u_{r}(r)\right|^{p}$, we deduce (3.14) by applying Lemma 3.2

Proposition 3.3. Let $\left\{r_{n}\right\} \subset(0,1],\left\{M_{n}\right\} \subset \mathbb{R}^{+}$two sequences with $r_{n} \downarrow 0$. Then, for $N \geq p+4 p /(p-1)$, there exists $u \in W^{1, p}\left(B_{1}\right)$, which is a semistable radially decreasing unbounded solution of a problem of the type (1.1), satisfying

$$
\left|u_{r}\left(r_{n}\right)\right| \geq M_{n} \quad \forall n \in \mathbb{N} .
$$

Proof. It is easily seen that for every sequences $\left\{r_{n}\right\} \subset(0,1],\left\{y_{n}\right\} \subset \mathbb{R}^{+}$, with $r_{n} \downarrow 0$, there exists a nonnegative function $h \in\left(C^{2} \cap L^{1}\right)(0,1]$ satisfying $h\left(r_{n}\right)=y_{n}$. Take $y_{n}=(N-1) M_{n}^{p} r_{n}^{N-2 \sqrt{\frac{N-1}{p-1}}-3}$ and apply Theorem 3.1 with this function $h$. It is clear, from the definition of $\Phi$, that $\Phi^{\prime}(r) \geq$ $r^{2} \sqrt{\frac{N-1}{p-1}} h(r), r \in(0,1]$. Hence

$$
(N-1) r_{n}^{N-3}\left|u_{r}\left(r_{n}\right)\right|^{p}=\Phi^{\prime}\left(r_{n}\right) \geq r_{n}^{2 \sqrt{\frac{N-1}{p-1}}} h\left(r_{n}\right)=r_{n}^{2 \sqrt{\frac{N-1}{p-1}}}(N-1) M_{n}^{p} r_{n}^{N-2 \sqrt{\frac{N-1}{p-1}}-3}
$$


and the proposition follows.

Corollary 3.4. Let $N \geq p+4 p /(p-1)$. There does not exist a function $\psi:(0,1] \rightarrow \mathbb{R}^{+}$with the following property: for every $u \in W^{1, p}\left(B_{1}\right)$ semistable radially decreasing solution of a problem of the type (1.1), there exist $C>0$ and $\epsilon \in(0,1]$ such that $\left|u_{r}(r)\right| \leq C \psi(r)$ for $r \in(0, \epsilon]$.

Proof. Suppose that such a function $\psi$ exists and consider the sequences $r_{n}=$ $1 / n, M_{n}=n \psi(1 / n)$. By the proposition above, there exists $u \in W^{1, p}\left(B_{1}\right)$, which is a semi-stable radially decreasing unbounded solution of a problem of the type (1.1), satisfying $\left|u_{r}(1 / n)\right| \geq n \psi(1 / n)$, a contradiction.

Proposition 3.5. Let $\left\{r_{n}\right\} \subset(0,1],\left\{M_{n}\right\} \subset \mathbb{R}^{+}$two sequences with $r_{n} \downarrow 0$. Then, for $N \geq p+4 p /(p-1)$, there exists $u \in W^{1, p}\left(B_{1}\right)$, which is a semistable radially decreasing unbounded solution of a problem of the type (1.1) with $g \geq 0$, satisfying

$$
\left|u_{r r}\left(r_{n}\right)\right| \geq M_{n} \forall n \in \mathbb{N} .
$$

Proof. Let $h \in C^{2}(0,1]$, increasing, satisfying $0 \leq h \leq 1$. Define $\Phi$ and $u_{r}$ as in Theorem 3.1. We claim that

i) $u$ is a semi-stable radially decreasing unbounded $W^{1, p}\left(B_{1}\right)$ solution of a problem of the type (1.1) with $g \geq 0$.

ii) $\left|u_{r}(r)\right| \leq D_{N, p} r^{-\frac{1}{p}\left(N-2 \sqrt{\frac{N-1}{p-1}}-2\right)}, \forall r \in(0,1]$, where $D_{N, p}$ only depends on $N$ and $p$.

iii) $-u_{r r}(r) \geq E_{N, p} r^{-\frac{1}{p}\left(N-2 \sqrt{\frac{N-1}{p-1}}-p-2\right)} h^{\prime}(r)-F_{N, p} r^{-\frac{1}{p}\left(N-2 \sqrt{\frac{N-1}{p-1}}+p-2\right)}$, $\forall r \in(0,1]$, where $E_{N, p}>0$ and $F_{N, p}$ only depend on $N$ and $p$.

Since $h$ is positive and increasing, then $\Phi^{\prime \prime}>0$. Hence $(N-1) r^{N-3}\left|u_{r}(r)\right|^{p}$ is increasing and so is

$$
r^{\frac{p(N-1)}{p-1}}\left|u_{r}(r)\right|^{p}=\left(-r^{N-1}\left|u_{r}(r)\right|^{p-2} u_{r}(r)\right)^{\frac{p}{p-1}} .
$$

This implies that $-r^{N-1}\left|u_{r}(r)\right|^{p-2} u_{r}(r)$ is increasing, which is is equivalent to the positiveness of $g$.

On account of $0 \leq h \leq 1$, we have $\Phi^{\prime}(r) \leq G_{N, p} r^{2 \sqrt{\frac{N-1}{p-1}}-1}$ in $(0,1]$, for a constant $G_{N, p}$ that only depends on $N$ and $p$. Hence, from the definition of $u_{r}$, we obtain ii).

From the positiveness of $h$ and $N \geq p+4 p /(p-1)$, we obtain $\Phi^{\prime \prime}(r) \geq$ $r^{2 \sqrt{\frac{N-1}{p-1}}} h^{\prime}(r)$ in $(0,1]$. On the other hand, from the definition of $u_{r}$ we have $\Phi^{\prime \prime}(r)=(N-1)\left[(N-3) r^{N-4}\left|u_{r}(r)\right|^{p}+p r^{N-3}\left|u_{r}(r)\right|^{p-2} u_{r}(r) u_{r r}(r)\right]$. Therefore, by $i i)$ and the previous inequality, we obtain iii).

Finally, it is easily seen that for every sequences $\left\{r_{n}\right\} \subset(0,1],\left\{y_{n}\right\} \subset \mathbb{R}^{+}$, with $r_{n} \downarrow 0$, there exists $h \in C^{2}(0,1]$, increasing, satisfying $0 \leq h \leq 1$ and 
$h^{\prime}\left(r_{n}\right)=y_{n}$. Take $y_{n}$ such that

$$
E_{N, p} r_{n}^{-\frac{1}{p}\left(N-2 \sqrt{\frac{N-1}{p-1}}-p-2\right)} y_{n}-F_{N, p} r_{n}^{-\frac{1}{p}\left(N-2 \sqrt{\frac{N-1}{p-1}}+p-2\right)}=M_{n}
$$

Applying iii) we deduce $-u_{r r}\left(r_{n}\right) \geq M_{n}$ and the proof is complete.

Corollary 3.6. Let $N \geq p+4 p /(p-1)$. There does not exist a function $\psi:(0,1] \rightarrow \mathbb{R}^{+}$with the following property: for every $u \in W^{1, p}\left(B_{1}\right)$ semistable radially decreasing solution of a problem of the type (1.1) with $g \geq 0$, there exist $C>0$ and $\epsilon \in(0,1]$ such that $\left|u_{r r}(r)\right| \leq C \psi(r)$ for $r \in(0, \epsilon]$.

Proof. Arguing as in Corollary 3.4 and using Proposition 3.5, we conclude the proof of the corollary.

Proposition 3.7. Let $\left\{r_{n}\right\} \subset(0,1],\left\{M_{n}\right\} \subset \mathbb{R}^{+}$two sequences with $r_{n} \downarrow 0$. Then, for $N \geq p+4 p /(p-1)$, there exists $u \in W^{1, p}\left(B_{1}\right)$, which is a semistable radially decreasing unbounded solution of a problem of the type (1.1) with $g, g^{\prime} \geq 0$, satisfying

$$
\left|u_{r r r}\left(r_{n}\right)\right| \geq M_{n} \forall n \in \mathbb{N}
$$

Lemma 3.8. For any dimension $N \geq p+4 p /(p-1)$, there exists $\epsilon_{N, p}>$ 0 with the following property: for every $h \in C^{2}(0,1] \cap C^{1}[0,1]$ satisfying $h(0)=0,0 \leq h^{\prime} \leq \epsilon_{N, p}$ and $h^{\prime \prime} \leq 0, u$ is a semi-stable radially decreasing unbounded $W^{1, p}\left(B_{1}\right)$ solution of a problem of the type (1.1) with $g, g^{\prime} \geq 0$ where $u_{r}$ is defined in terms of $h$ as in Theorem 3.1.

Proof. Similarly as in the proof of Proposition 3.5 (item $i)$ ), $h^{\prime} \geq 0$ implies that $u$ is a semi-stable radially decreasing unbounded $W^{1, p}\left(B_{1}\right)$ solution of a problem of the type (1.1) with $g \geq 0$.

On the other hand, from the definition of $\Phi$ and $u_{r}$ it follows easily that

$$
\begin{aligned}
u_{r} & =-\left[(N-1)^{-1} r^{-N+3} \Phi^{\prime}\right]^{\frac{1}{p}} \\
& =-r^{-\frac{1}{p}\left(N-2 \sqrt{\frac{N-1}{p-1}}-2\right)}\left[\frac{2+2 \int_{0}^{r} h(s) d s}{\sqrt{(N-1)(p-1)}}+\frac{r h(r)}{N-1}\right]^{\frac{1}{p}} .
\end{aligned}
$$

Put this last expression in the form $u_{r}=-r^{-\frac{1}{p}\left(N-2 \sqrt{\frac{N-1}{p-1}}-2\right)} \varphi(r)$, where $\varphi(r)$ (and of course $u_{r}$ ) depends on $h$. Now consider the set

$$
X=\left\{h \in C^{2}(0,1] \cap C^{1}[0,1]: h(0)=0,0 \leq h^{\prime}, h^{\prime \prime} \leq 0\right\},
$$

and the norm $\|h\|_{X}=\left\|h^{\prime}\right\|_{L^{\infty}(0,1)}$. Taking $\|h\|_{X} \rightarrow 0$, we have

$$
\begin{gathered}
\lim _{\|h\|_{X \rightarrow 0}} \varphi=\left[\frac{2}{\sqrt{(N-1)(p-1)}}\right]^{\frac{1}{p}}, \lim _{\|h\|_{X \rightarrow 0}} \varphi^{\prime}=0, \\
\lim _{\|h\|_{X} \rightarrow 0}\left(\varphi^{\prime \prime}-\frac{r h^{\prime \prime}}{p(N-1) \varphi^{p-1}}\right)=0,
\end{gathered}
$$


where all the limits are taken uniformly in $r \in(0,1]$. On the other hand, it is easy to check that

$$
\begin{aligned}
\frac{g^{\prime}(u) r^{2}}{p-1}= & -\left|u_{r}\right|^{p-2}\left[(p-2)\left(\frac{r u_{r r}}{u_{r}}\right)^{2}+(N-1)\left(\frac{r u_{r r}}{u_{r}}\right)\right. \\
& \left.+\left(\frac{r^{2} u_{r r r}}{u_{r}}\right)-\frac{N-1}{p-1}\right] \\
= & -\left|u_{r}\right|^{p-2}\left[(p-2)\left(\frac{r \varphi^{\prime}}{\varphi}\right)^{2}+\frac{r^{2} \varphi^{\prime \prime}}{\varphi}\right. \\
& +\frac{1}{p}\left((2-p) N+4(p-1) \sqrt{\frac{N-1}{p-1}}+3 p-4\right)\left(\frac{r \varphi^{\prime}}{\varphi}\right) \\
& -\frac{1}{p^{2}}\left(N-2 \sqrt{\frac{N-1}{p-1}}-2\right)\left(N+2(p-1) \sqrt{\frac{N-1}{p-1}}-2\right) \\
& \left.-\frac{N-1}{p-1}\right] .
\end{aligned}
$$

Hence, from (3.15), we can assert that, for $h \in X$ with small $\|h\|_{X}$, $r^{2} g^{\prime}(u) \geq 0$ in $(0,1]$ and the lemma follows.

Proof of Proposition 3.7. We follow the notation used in the previous lemma. From (3.15), we deduce that

$$
\lim _{\|h\|_{X} \rightarrow 0}\left(r^{\frac{1}{p}\left(N-2 \sqrt{\frac{N-1}{p-1}}+2 p-2\right)} u_{r r r}+\frac{r^{3} h^{\prime \prime}}{p(N-1) \varphi^{p-1}}\right)=\sigma
$$

uniformly in $r \in(0,1]$, where

$$
\sigma=-2^{\frac{1}{p}} \frac{\left[\left(-\frac{1}{p}\right)\left(N-2 \sqrt{\frac{N-1}{p-1}}-2\right)\right]\left[\left(-\frac{1}{p}\right)\left(N-2 \sqrt{\frac{N-1}{p-1}}+p-2\right)\right]}{(\sqrt{(N-1)(p-1)})^{\frac{1}{p}}}<0 .
$$

Then, taking $\epsilon_{N, p}^{\prime}>0$ sufficient small (possibly less than $\epsilon_{N, p}$ ), we have that

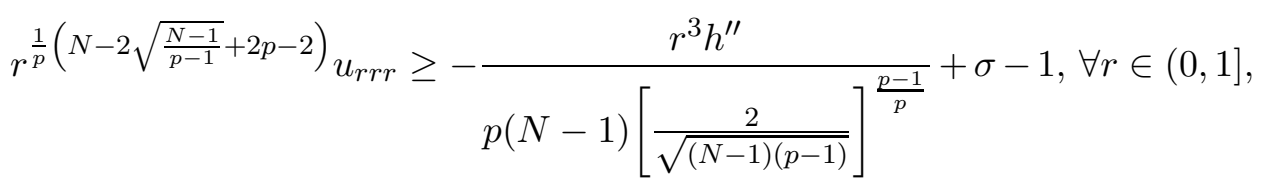

for $\|h\|_{X} \leq \epsilon_{N, p}^{\prime}$.

Finally, it is easily seen that for every sequences $\left\{r_{n}\right\} \subset(0,1],\left\{y_{n}\right\} \subset \mathbb{R}^{+}$, with $r_{n} \downarrow 0$, there exists $h \in X$, with $\|h\|_{X} \leq \epsilon_{N, p}^{\prime}$, satisfying $h^{\prime \prime}\left(r_{n}\right)=-y_{n}$. 
Take $y_{n}$ such that

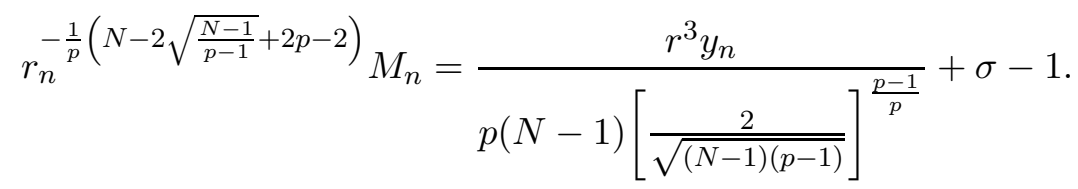

Applying the above inequality, we obtain $u_{r r r}\left(r_{n}\right) \geq M_{n}$ and the proof is complete.

Corollary 3.9. Let $N \geq p+4 p /(p-1)$. There does not exist a function $\psi$ : $(0,1] \rightarrow \mathbb{R}^{+}$with the following property: for every $u \in W^{1, p}\left(B_{1}\right)$ semi-stable radially decreasing solution of a problem of the type (1.1) with $g, g^{\prime} \geq 0$, there exist $C>0$ and $\epsilon \in(0,1]$ such that $\left|u_{r r r}(r)\right| \leq C \psi(r)$ for $r \in(0, \epsilon]$.

Proof. Applying Proposition [3.7. this follows by the same method as in Corollaries 3.4 and 3.6 .

\section{REFERENCES}

[1] Haim Brezis and Juan Luis Vázquez. Blow-up solutions of some nonlinear elliptic problems. Rev. Mat. Univ. Complut. Madrid, 10(2):443-469, 1997.

[2] Xavier Cabré. Extremal solutions and instantaneous complete blow-up for elliptic and parabolic problems. In Perspectives in nonlinear partial differential equations, volume 446 of Contemp. Math., pages 159-174. Amer. Math. Soc., Providence, RI, 2007.

[3] Xavier Cabré, Antonio Capella, and Manel Sanchón. Regularity of radial minimizers of reaction equations involving the p-Laplacian. Calc. Var. Partial Differential Equations, 34(4):475-494, 2009.

[4] Xavier Cabré and Manel Sanchón. Semi-stable and extremal solutions of reaction equations involving the $p$-Laplacian. Commun. Pure Appl. Anal., 6(1):43-67, 2007.

[5] J. Dávila. Singular solutions of semi-linear elliptic problems. In Handbook of differential equations: stationary partial differential equations. Vol. VI, Handb. Differ. Equ., pages 83-176. Elsevier/North-Holland, Amsterdam, 2008.

[6] J. Dávila and L. Dupaigne. Perturbing singular solutions of the Gelfand problem. Commun. Contemp. Math., 9(5):639-680, 2007.

[7] Juan Dávila, Louis Dupaigne, Ignacio Guerra, and Marcelo Montenegro. Stable solutions for the bilaplacian with exponential nonlinearity. SIAM J. Math. Anal., 39(2):565-592, 2007.

[8] Juan Dávila, Louis Dupaigne, and Marcelo Montenegro. The extremal solution of a boundary reaction problem. Commun. Pure Appl. Anal., 7(4):795-817, 2008.

[9] S. Eidelman and Y. Eidelman. On regularity of the extremal solution of the Dirichlet problem for some semilinear elliptic equations of the second order. Houston J. Math., 31(3):957-960, 2005.

[10] Pierpaolo Esposito. Compactness of a nonlinear eigenvalue problem with a singular nonlinearity. Commun. Contemp. Math., 10(1):17-45, 2008.

[11] J. García Azorero, I. Peral Alonso, and J.-P. Puel. Quasilinear problems with exponential growth in the reaction term. Nonlinear Anal., 22(4):481-498, 1994.

[12] Nassif Ghoussoub and Yujin Guo. On the partial differential equations of electrostatic MEMS devices: stationary case. SIAM J. Math. Anal., 38(5):1423-1449 (electronic), $2006 / 07$.

[13] D. D. Joseph and T. S. Lundgren. Quasilinear Dirichlet problems driven by positive sources. Arch. Rational Mech. Anal., 49:241-269, 1972/73.

[14] Gueorgui Nedev. Regularity of the extremal solution of semilinear elliptic equations. C. R. Acad. Sci. Paris Sér. I Math., 330(11):997-1002, 2000. 
[15] Manel Sanchón. Boundedness of the extremal solution of some $p$-Laplacian problems. Nonlinear Anal., 67(1):281-294, 2007.

[16] Salvador Villegas. Sharp estimates for semi-stable radial solutions of semilinear elliptic equations. J. Funct. Anal., 262(7):3394-3408, 2012.

[17] Salvador Villegas. Boundedness of extremal solutions in dimension 4. Adv. Math., 235:126-133, 2013

Departamento de Análisis Matemático, Universidad de Granada, 18071 Granada, SPAIN.

E-mail address: mnavarro_2@ugr.es and svillega@ugr.es 